\title{
Growth Measurement of Arabidopsis in 2.5D from a High Throughput Phenotyping Platform
}

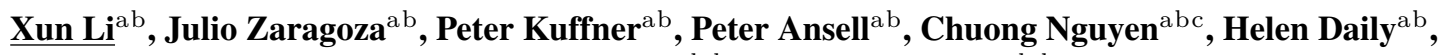 \\ Robert Furbank $^{\text {bd }}$ and Xavier Sirault ${ }^{\text {abd }}$ \\ ${ }^{a}$ High Resolution Plant Phenomics Centre, Canberra, ACT, Australia \\ ${ }^{\mathrm{b}}$ CSIRO Agriculture Flagship, Cnr Clunies Ross St and Barry Dr, Canberra, ACT Australia \\ ${ }^{\mathrm{c}}$ Australian National University, Centre of Excellence for Robotics Vision, Canberra, ACT, Australia \\ ${ }^{\mathrm{d}}$ Australian National University, Centre of Excellence for Translational Photosynthesis, Canberra, ACT, \\ Australia \\ Email: $\underline{x u n .1 i @ \text { csiro.au }}$
}

\begin{abstract}
The last few years have seen an increasing interest in the development of high throughput phenotyping platforms (HTP) that allow the automated measurement of plant growth and structure. These platforms have utilised various imaging technologies, including fluorescence imaging, thermal imaging, and visible imaging. Since plants are structurally complex and inherently three-dimensional (3D), 3D imaging and reconstruction approaches have distinct advantages over 2D imaging when it comes to quantifying structural information (such as leaf angle distribution, leaf area etc.).

High throughput phenotyping platforms, when combined with 3D image analysis, enable researchers to investigate complex functional traits related to plant structure, including responses to external and internal signals or perturbations. Moreover, image-based reconstruction techniques are able to produce complex structural models of both large and small individual plants, which retain colour information and are topologically coherent.

In this paper, we present a computational workflow for analysing the growth of Arabidopsis thaliana rosettes over time using stereo reconstruction. Stereo reconstruction for plant phenotyping generally includes data collection and point cloud generation. In our approach, we not only use stereo image pairs to generate point clouds of the plants but also reconstruct mesh surfaces for further analysis.
\end{abstract}

By applying a semi-global matching algorithm, 2.5D point clouds of Arabidopsis growing in trays with multiple plants were generated. Point clouds were then filtered and segmented in order to isolate single plants using clustering methods. A variety of surface reconstruction methods were then used to generate meshes from the point clouds. These meshes were analysed to quantify the plant dimensions and surface area in three dimensions. The segmentation was stable over time and was used to generate a time series for the growth of the individual plants over time.

The advantages of such a stereo reconstruction system are: i) the system is able to construct realistic point clouds and meshes of the scene, ii) the processing pipeline is computationally efficient, iii) it allows stereo reconstruction and segmentation of individual plants in trays of 20 plants each for high throughput analysis. The overall approach proved useful in quantifying morphometric parameters in 3D for a set of Arabidopsis accessions and relating plant structure to plant function.

Keywords: Stereoscopic reconstruction, 3D mesh, Arabidopsis, plant phenotyping, semi-global matching, point-cloud filtering 


\section{INTRODUCTION}

Automated methods for measuring plant growth and performance in high throughput are necessary to understand the genetics of plant development under different environments. These observations when linked to genotype and environment information are a powerful tool to dissect the genetic architecture of traits. By capturing the development of plants at high spatial and temporal resolutions, researchers can investigate plant growth responses to external and internal signals non-destructively (Furbank and Tester, 2011). Since plants are structurally complex and three dimensional in nature, 2D imaging methods based on single cameras suffer from inaccuracies. For instance when using projected area as a surrogate for biomass or total plant surface area, leaf areas are calculated without taking into account leaf angles. In contrast, one of the advantages of using 3D meshes of the plants over 2D analysis is that it is more robust to leaf movement when determining leaf area on a single plant basis. Moreover, parameters such as surface normal can be used to assess metrics such as the orientation of single leaves. The drawback is that these approaches are often more time consuming. They often require multiple cameras or multiple images captured from several different poses, and an accurate knowledge of the camera intrinsic and extrinsic parameters.

In this paper, we present a new computational approach applied to the analysis of Arabidopsis thaliana rosette growth over time using stereo reconstruction. Our approach relies on the integration of a number of visionbased 3D reconstruction methods to enable the determination of characteristics of plants grown in seedling trays. The paper is constructed as follows: Section 2 describes the background information and related work. Our methodology is described in Section 3 and experimental results are presented in Section 4. Concluding remarks and future approaches are discussed in Section 5.

\section{BACKGROUND}

Imaging technologies have been integrated into high-throughput phenotyping platforms to probe various aspects of plant structure and function (Granier et al., 2006; Paproki et al., 2012). Compared with other approaches, using images is amenable to automated quantification of individual plants. In particular, parameters regarding plant growth, yield and stress responses can be more readily identified. At present, imaging techniques for plant phenotyping primarily include fluorescence imaging, thermal infrared imaging, visible imaging, spectroscopy and other techniques (MRI, PET and CT). As visible imaging is low cost, it is available in commercial phenotyping platforms. It is primarily used to measure aspects of plant architecture including projected surface as a surrogate for biomass, colour information to study profiles of leaf senescence, and structural information (Li et al., 2014; Ivanov et al., 1995).

At present, 3D reconstructions for phenotyping platforms mainly focus on manipulating point clouds obtained from 3D laser scanning technology in both field and laboratory environments. However, laser scanning produces sparse point clouds, without color information, that are difficult to filter for identifying plant structural characteristics. Thus, the use of passive sensors (e.g. stereo cameras) have become increasingly important for plant phenotyping (Lou et al., 2014; Kumar et al., 2014). Stereo reconstruction is achieved by capturing images from slightly different angles. The disparity between stereo pairs allows inference of depth information. Based on the optimization method, stereo matching algorithms generally fall into two groups: local and global matching. Local methods such as block matching use a sliding window to find the minimum matching cost, like the sum of absolute differences (Sabater et al., 2011). However, it implicitly assumes that all pixels within the window are at the same distance from the camera, leading to problems with object boundaries and discontinuous regions. Global methods, on the other hand, make explicit smoothness assumptions of the disparity map and minimise the cost function on a global basis, but are generally computationally expensive. Classic algorithms of this type are Dynamic Programming (Kim et al., 2005), Graph Cut (Kolmogorov and Zabih, 2001) and Belief Propagation (Felzenszwalb and Huttenlocher, 2004). Semi-global Matching (Hirschmüller, 2008) successfully combines the benefits from both groups and the algorithm is able to perform accurate image matching at lower computational cost. Based on the disparity map generated by stereo matching and camera calibration information, a point cloud can be generated by performing re-projection from 2D pixels to 3D points.

The methods for surface reconstruction from point cloud can be categorized into three groups: region growing, computational geometry and algebraic methods. Region growing algorithms use a progressive approach to efficiently produce a polygonal surface. The implementation of the Greedy Projection in Rusu and Cousins (2011) has been popular in this field. Among the computational geometry methods, Delaunay Triangulation has been commonly used to interpolate the points and to try and fit the mesh into existing datasets. Power Crust 
(Amenta et al., 2001) is also a well-established method among this group. Unfortunately, the computational geometry approaches are sensitive to noise or datasets with varying point density. The final group generally uses an implicit function to approximate the surface normal, among which Hoppe's method (Hoppe et al., 1992), MPU (Ohtake et al., 2005) and Poisson reconstruction (Kazhdan et al., 2006) are favourites. These algorithms have the main advantage of being robust to noise as well as varying point density. Given these various advantages and disadvantages, our approach focused on combining approaches from the first and third groups to reconstruct the surface of individual A. thaliana plants.

\section{METHOD}

In this section we describe the approach used to build an automated stereo reconstruction pipeline to analyse the growth of $A$. thaliana rosettes over time using top down images from a stereo camera pair in a phenotyping platform. The platform, namely "TrayScan", enclosed 3 types of cameras mounted overhead in three stations: a stereo RGB camera station with resolution of 5 mega pixels and a baseline of $62 \mathrm{~mm}$, a FLIR infrared imaging station, and a pulse modulated chlorophyll fluorescence imaging station. We introduce our acquisition methodology and describe the reconstruction process by using one tray with 20 individual pots of $A$. thaliana at one time point (Arabidopsis-thaliana_Tray_0001-date-2014-07-17T11-48-30-round-001-003). The processing pipeline described here was in fact used to process a dataset composed of 60 trays at 20 time points.

\subsection{Stereo Reconstruction by Using Semi-global Matching}

Our stereo reconstruction module is based on the semi-global matching method with the associated cost function given in Eq. 1:

$$
E(D)=\sum_{p}\left(C\left(p, D_{p}\right)+\sum_{q \in N_{p}} P_{1} T\left[\left|D_{p}-D_{q}\right|=1\right]+\sum_{q \in N_{p}} P_{2} T\left[\left|D_{p}-D_{q}\right|>1\right]\right) .
$$

where $E(D)$ is the global energy function for disparity map $D . C\left(p, D_{p}\right)$ is the matching cost of pixel point $p$ with the depth $D_{p}$. The second and third terms are the smoothing constraints. $N_{p}$ is the neighbourhood of pixel $p . q$ represents the neighbourhood pixels of $p$ in the image. $P_{1}$ is the penalty on the disparity change by \pm 1 between neighbour pixels. $P_{2}$ is the penalty function for the disparity change by more than 1 between neighbourhood pixels. $T[\cdot]$ is a binary function giving 1 if the argument is true and 0 otherwise. Minimizing the cost function is solved by performing multiple 1D minimization, and aggregating costs on multiple paths according to Hirschmüller (2008).

After images were rectified, pairs of stereo images were matched using semi-global matching. The disparity map was generated along with the 3D point cloud for the whole tray. It is noted that stereo anaglyphs (Dubois, 2001) were generated in order to set the proper disparity range parameter for the matching algorithm. The three dimensional accuracy of the point cloud generated from the pipeline was verified by using a test tray without plants in it. It reached millimetre level accuracy in all three dimensions. Our test shows that camera resolution is closely related to the density of the point cloud, thus affecting the quality of the mesh with related metrics (e.g. surface areas). Therefore high resolution camera is essential. Results for the sample tray are presented in Fig. 1(a).

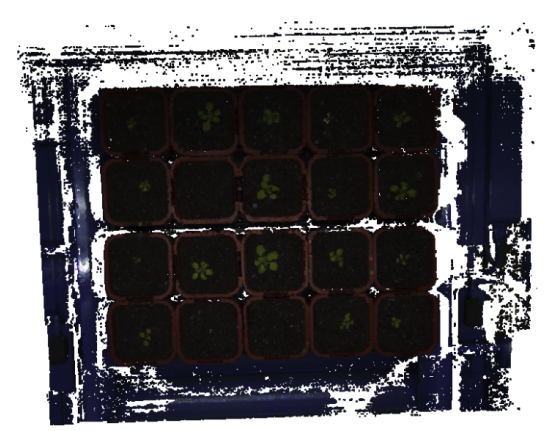

(a) Point cloud $(\mathbf{P})$ of the sample tray

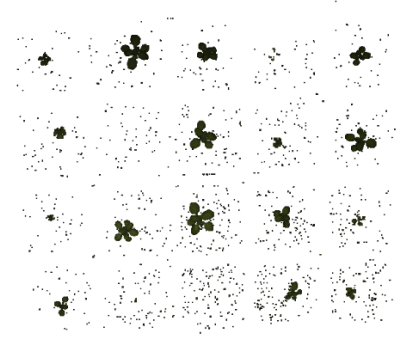

(b) Point cloud results after the green area (c) segmentation process $(\mathbf{F})$.

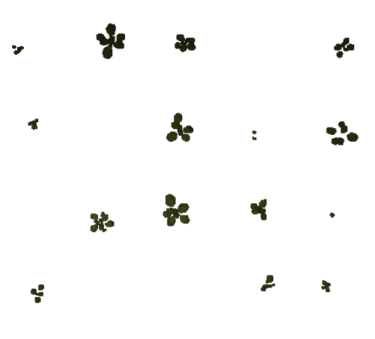

(c) Point cloud filtering results after noise filtering $\left(\mathbf{F}^{\prime}\right)$.

Figure 1.The point cloud with filtering processes for a sample tray. 


\subsection{Plant Segmentation}

After generating the initial point cloud, we developed a plant segmentation module to retain only the 3D points of interest (i.e., the plants) while removing the background points. This step involves two processes: green area segmentation and noise filtering. Following this step, each plant was individually extracted for further analysis.

Green Area Segmentation. The green area segmentation process detected the green area of the plants as a function of the difference between the red and green channels of the 2.5D point (Meyer and Neto, 2008). This approach was effective for eliminating the $2.5 \mathrm{D}$ points that corresponded to elements like pots, soil or trays and were present in the input images (Fig. 1(b)). Since each point $\mathbf{p}_{i}$ from a point cloud $\mathbf{P}=\left\{\mathbf{p}_{1}, \mathbf{p}_{2}, \cdots, \mathbf{p}_{N}\right\}$ is a 6-tuple $\mathbf{p}_{i}=\left\langle x_{i}, y_{i}, z_{i}, r_{i}, g_{i}, b_{i}\right\rangle$, where $(x, y, z)$ represent the spatial position of the point and $(r, g, b)$ represent the colour of the point, we filtered the point cloud $\mathbf{F}$ by applying the following equation:

$$
\mathbf{F}=\mathbf{P} \backslash\left\{\mathbf{p}_{i}\right\} \text { if }\left(E x G_{i}-E x R_{i}\right)>t \text { for } i=1,2, \cdots, N,
$$

where $E x G_{i}=2 \times g_{i}-r_{i}-b_{i}$ and $E x R_{i}=1.4 \times r_{i}-b_{i}$ and $t$ is a threshold value set to 10 in this paper.

Noise Filtering. Residual noise due to points that have a colour that is similar to that of the plants or induced by lighting variations can be eliminated by tuning the value of the threshold $t$ in Eq. 2. However, such an approach can compromise the robustness of the filtering process since $t$ might need to be tuned for each specific case. In this paper we adopted a statistical outlier removal process to eliminate these spurious points. For each point $\mathbf{p}_{i}$ in $\mathbf{F}$ we selected a set $\mathbf{Q}^{i}=\left\{\mathbf{p}_{1}^{i}, \mathbf{p}_{2}^{i}, \ldots, \mathbf{p}_{K}^{i}\right\} \subset \mathbf{F}$, which are the $K$ nearest neighbours of $\mathbf{p}_{i}$, and calculate this set's mean distance $\mu_{i}$ and standard deviation $\sigma_{i}$ to $\mathbf{p}_{i}$ as $\mu_{i}=\frac{\sum_{\mathbf{p}_{j}^{i} \in \mathbf{Q}^{i}} \sqrt{\left(x_{i}-x_{j}^{i}\right)^{2}+\left(y_{i}-y_{j}^{i}\right)^{2}+\left(z_{i}-z_{j}^{i}\right)^{2}}}{K}$ and $\sigma_{i}=\sqrt{\frac{\sum_{\mathbf{p}_{j}^{i} \in \mathbf{Q}^{i}}\left(x_{j}^{i}-\mu_{i}\right)^{2}+\left(y_{j}^{i}-\mu_{i}\right)^{2}+\left(z_{j}^{i}-\mu_{i}\right)^{2}}{K}}$. Point $\mathbf{p}_{i}$ is denoted as outlier if $\mu_{i}>\mu+\kappa * \sigma$, where $\mu=\sum_{i=1}^{|\mathbf{F}|} \mu_{i} /|\mathbf{F}|, \sigma=\sum_{i=1}^{|\mathbf{F}|} \sigma_{i} /|\mathbf{F}|$ and $\kappa$ is a free multiplier parameter that is set to 1 in our experiments. If a point $\mathbf{p}_{i}$ is catalogued as an outlier, it is then removed from F. The result from this noise filtering process is a filtered set $\mathbf{F}^{\prime}$ of $2.5 \mathrm{D}$ points. Fig. 1(c) presents an example result of this process. The initial point cloud contained 55224 points and 37402 points after filtering.

Point Cloud Clustering. After the noise was removed from the point cloud, the next step was to extract each one of the reconstructed Arabidopsis plants in order to perform individual plant analysis. A Euclidean clustering processing method was used to find and segment the individual object point clusters (i.e., the plants) that were present in the point cloud. The Euclidean clustering process is presented in Alg. 1.

The purpose of this algorithm was to generate small plant clusters from the 3D points that were distinct from each other. This simple approach is effective at identifying each plant that was previously shown in Fig. 1(c). Fig. 2(a) presents a detailed result for one of these plants after clustering. As can be seen from these images, by using our proposed plant segmentation approach, each plant has been successfully filtered and separated from the rest of the initially noisy point cloud.

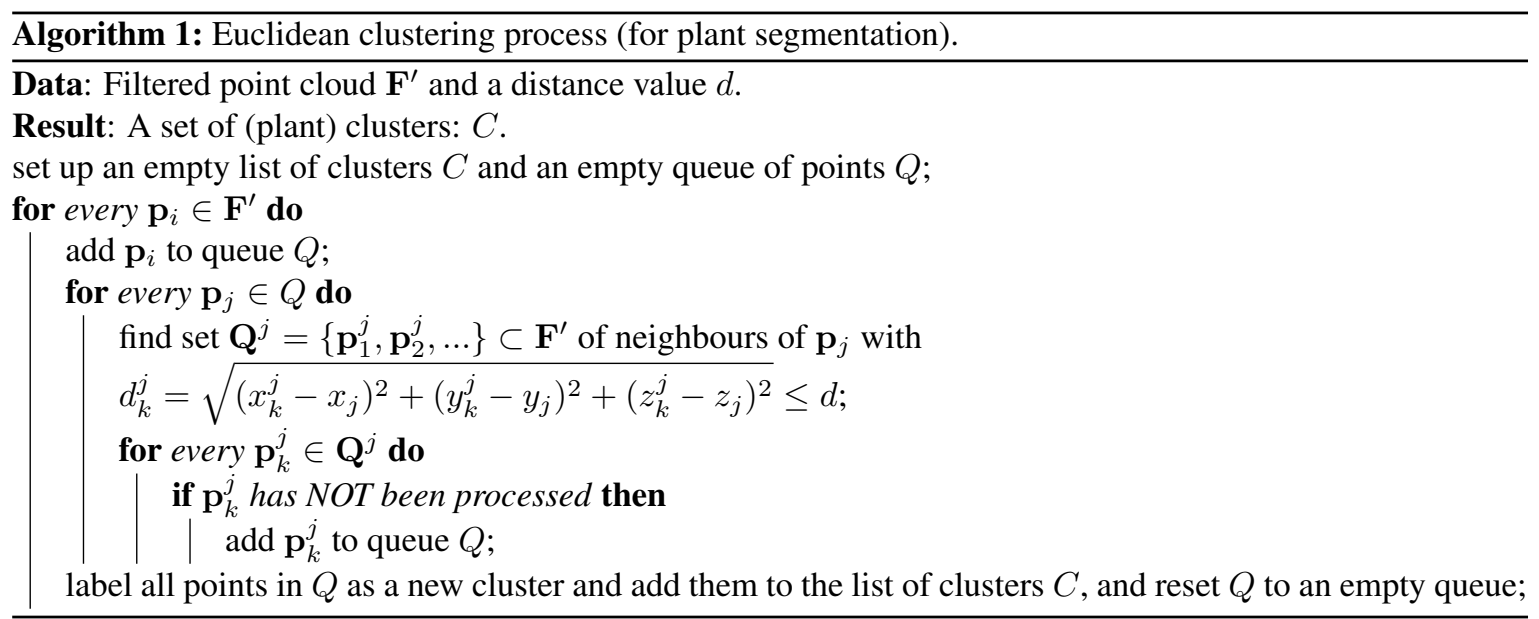




\subsection{Surface Reconstruction and Mesh Generation}

The next step was to generate surface meshes based on individual plant point clouds. The mesh generation module consists of two parts: a combination of the Greedy Projection and a hole filling algorithm followed by a Poisson Reconstruction method. Greedy projection keeps a list of points and grows a mesh by adding new triangles until all possible points are connected. The module also contains a hole filling function. As observed on Fig. 2(b), the Greedy Projection method constrained the mesh to the original point cloud (4896 vertices as 4896 points) by connecting only the existing points. The hole filling algorithm generated new faces without adding extra vertices to the original point cloud (Fig. 2(c)).

The second part of the mesh generation module includes the Poisson Reconstruction method. This algorithm requires oriented points to serve as samples of the solid boundary so that the gradient of the computed implicit function can be used to approximate the normal field of the boundary. Therefore, a normal estimation process was carried out before applying the Poisson Reconstruction from these oriented points. One characteristic of Poisson Reconstruction is that it produces a watertight surface, which is not exactly suitable for our scenario where leaves are presented in pieces and normally separated by gaps (Fig. 2(d)). Therefore, we applied the surface trimming algorithm developed by Kazhdan and Hoppe (2013) to the workflow for pruning the surface according to the sampling density of the point cloud. As shown in Fig. 2(e), the Poisson reconstruction algorithm generated new vertices and new faces while creating a smoother surface when compared to that of the Greedy Projection (Fig. 2(b)). One drawback of this approach is that, for non-watertight surfaces, finding the right parameters to trim the mesh remains a challenging task. Here we set the value for this specific scenario based on repeated tests. In a final step we derived parameters such as total surface areas and surface normals (Fig. 2(f)) to determine the angular distributions of the leaves.
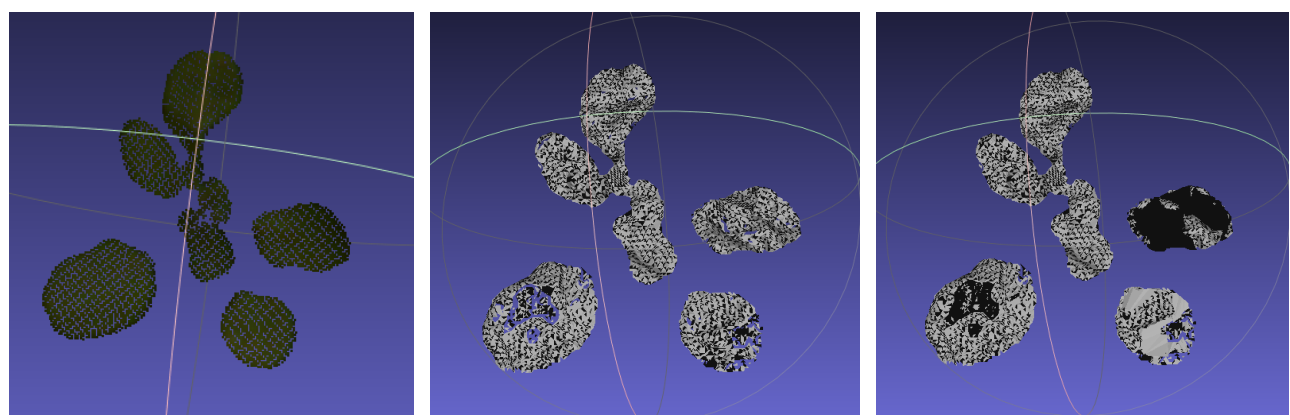

(a) Point cloud for Arabidops
of Pot 8 in the sample tray.

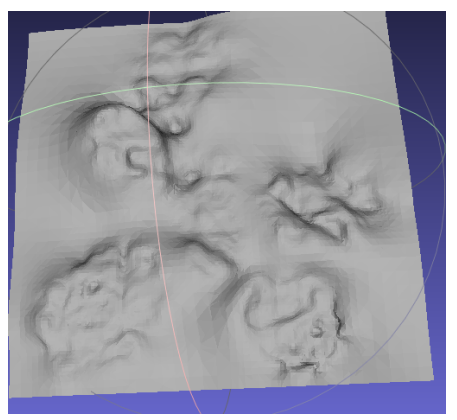
jection: 8428 faces and 4896 vertices.

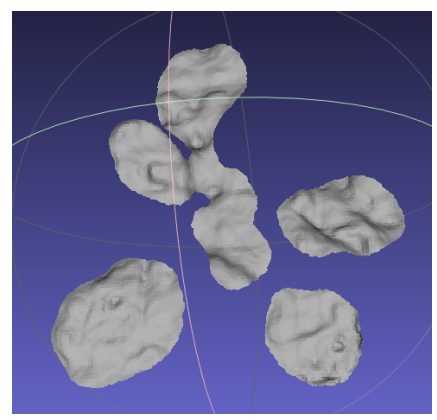

(d) 3D mesh after Poisson reconstruction: 25595 faces and 12885 vertices. (e) Trimmed Poisson reconstruction:
19800 faces and 10726 vertices. (c) 3D mesh with filled holes: 8872 faces and 4896 vertices.

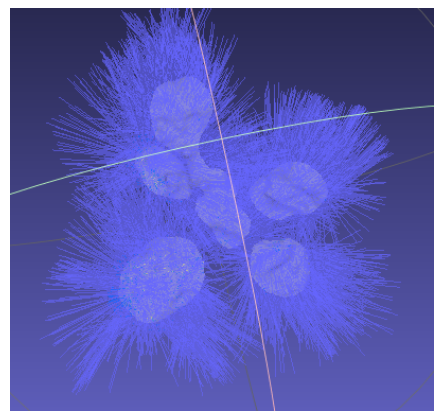

(f) The surface normals of the reconstructed mesh.

Figure 2.The 3D surface reconstruction process for Arabidopsis plants.

\section{EXPERIMENTS AND RESULTS}

The experiment for A. thaliana rosettes was designed as follows: a sample tray, part of a larger experiment (60 trays) in which 408 lines of a bi-parental (Bay-0 x Shahdara) mapping population of $A$. thaliana (plus the parents), was grown in a Debco potting mix in $5 \mathrm{~cm}$ pots in trays of 20 randomised plants. The plants were 
Table 1.Measured properties of the sample tray (unit: $\mathrm{mm}$, except for the Area: $\mathrm{mm}^{2}$ )

\begin{tabular}{|r|ccccccc|}
\hline Pot ID & x_center & y_center & z_center & x_length & y_length & z_length & Area \\
\hline 1 & -111.10 & -113.65 & 389.98 & 7.62 & 5.94 & 2.64 & 15.43 \\
2 & -35.22 & -120.35 & 393.79 & 22.17 & 27.21 & 5.11 & 237.95 \\
$\ldots$ & & & & & & & \\
19 & 98.01 & 89.27 & 392.15 & 11.04 & 14.11 & 3.69 & 46.18 \\
20 & 149.36 & 92.74 & 394.26 & 6.07 & 8.55 & 3.14 & 24.50 \\
\hline
\end{tabular}

grown in growth chambers and were scanned every weekday in a HTP phenotyping platform "TrayScan" over one month.

Metrics. After generating individual meshes, the plant's central position in 3D $\left(\mathrm{X}_{\text {center }}, \mathrm{Y}_{\text {center }}\right.$ and $\left.\mathrm{Z}_{\text {center }}\right)$ with respect to the fixed imaging system, the plant's size in terms of length, width, height and most importantly surface area were measured and quantified. The surface area was calculated by summing the areas of each triangle in the mesh. The result of the sample tray for captured plants (Arabidopsis-thaliana_Tray_0001-date2014-07-17T11-48-30-round-001-003) is shown in Table 1.

Application of the Pipeline to Characterising the Dynamics of Plant Development. In A. Thaliana Tray 0001, the automated workflow processed data from 20 time points (period 30/06/2014 to 25/07/2014). The dynamics of plant development was analysed by aggregating the surface areas of the same plant over time, as shown in Fig 3(a). The processing pipeline runs efficiently on a Dell Precision T7910 machine: it takes 90 to 180 seconds to process one tray at one time point. Thus the processing of 20 time points for one tray takes less than an hour. The accuracy of the surface area measurement depends on the mesh quality will be evaluated in future research using the scanned dissected leaves. The experimental setting is shown in Fig 3(b).

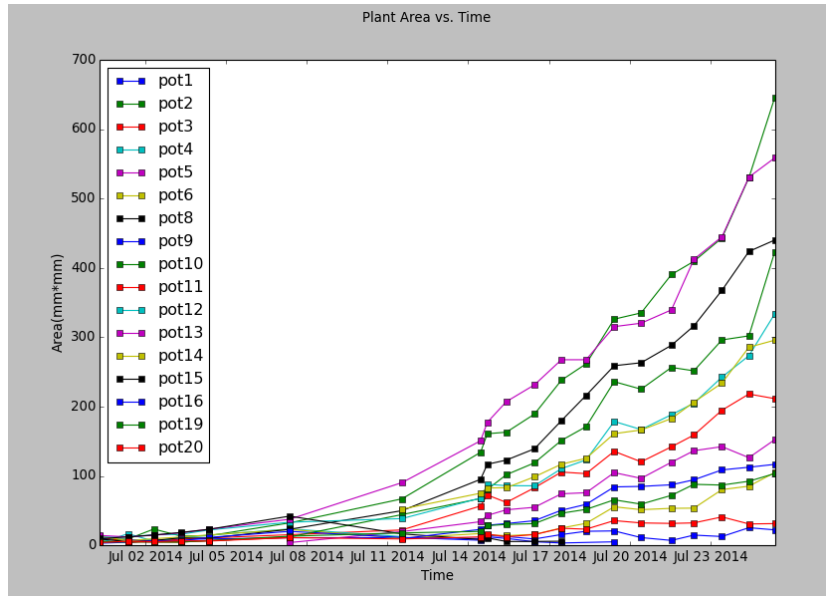

(a) Growth curve of the surface area for pots 1-20 from A. Thaliana Tray 0001 for the period $30 / 06 / 2014$ to $25 / 07 / 2014$.

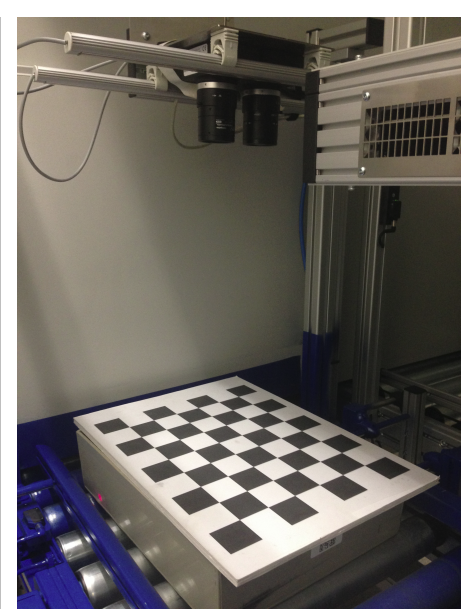

(b) Experimental Setting.

Figure 3.Growth curve and its experimental setting

\section{CONCLUSIONS}

In this paper, we presented an automated stereo reconstruction pipeline for analysing plant growth in HTP. The pipeline integrates various computer vision techniques (including stereo-matching, point cloud processing, surface reconstruction) in a single workflow. The workflow was tested using a dataset consisting of 60 trays with 20 time points. Results show that the system quantifies morphometric parameters in 3D for a set of $A$. thaliana accessions.

Currently, the workflow depends on an overhead stereo camera pair for 3D reconstruction, which is inherently fragile when leaves are occluded. More camera views will be added to the system to obtain more comprehen- 
sive information. Meanwhile, plant segmentation module will be further developed to deal with overlapping areas in the stereo images. Currently attributes such as surface area are calculated at the whole-plant level. Future work will focus on leaf segmentation based on the reconstructed mesh and extracting attributes at single leaf level. A system upgrade will be performed in the future to adopt higher resolution cameras, which will enable the pipeline to process other species, especially the ones with thin leaves.

\section{REFERENCES}

Amenta, N., S. Choi, and R. K. Kolluri (2001). The power crust. In Proceedings of the sixth ACM symposium on Solid modeling and applications, pp. 249-266. ACM.

Dubois, E. (2001). A projection method to generate anaglyph stereo images. In ICASSP 'O1, Volume 3, pp. 1661-1664.

Felzenszwalb, P. and D. Huttenlocher (2004, June). Efficient belief propagation for early vision. In CVPR 2004, Volume 1, pp. 261-268.

Furbank, R. T. and M. Tester (2011). Phenomics technologies to relieve the phenotyping bottleneck. Trends in Plant Science 16(12), 635 - 644.

Granier, C., L. Aguirrezabal, K. Chenu, S. J. Cookson, M. Dauzat, P. Hamard, J.-J. Thioux, G. Rolland, S. Bouchier-Combaud, A. Lebaudy, B. Muller, T. Simonneau, and F. Tardieu (2006). Phenopsis, an automated platform for reproducible phenotyping of plant responses to soil water deficit in arabidopsis thaliana. New Phytologist 169(3), 623-635.

Hirschmüller, H. (2008, Feb). Stereo processing by semiglobal matching and mutual information. TPAMI 30(2), 328-341.

Hoppe, H., T. DeRose, T. Duchamp, J. McDonald, and W. Stuetzle (1992). Surface reconstruction from unorganized points. In Proceedings of the 19th Annual Conference on Computer Graphics and Interactive Techniques, SIGGRAPH '92, New York, NY, USA, pp. 71-78. ACM.

Ivanov, N., P. Boissard, M. Chapron, and B. Andrieu (1995). Computer stereo plotting for 3D reconstruction of a maize canopy. Agricultural and Forest Meteorology 75(1-3), 85-102.

Kazhdan, M., M. Bolitho, and H. Hoppe (2006). Poisson surface reconstruction. In Proceedings of the fourth Eurographics symposium on Geometry processing, Volume 7.

Kazhdan, M. and H. Hoppe (2013, July). Screened poisson surface reconstruction. ACM ToG 32(3), 1-13.

Kim, J. C., K. M. Lee, B. T. Choi, and S. U. Lee (2005, June). A dense stereo matching using two-pass dynamic programming with generalized ground control points. In C. Schmid, S. Soatto, and C. Tomasi (Eds.), CVPR 2005, Volume 2, pp. 1075-1082. IEEE.

Kolmogorov, V. and R. Zabih (2001). Computing visual correspondence with occlusions using graph cuts. In ICCV 2001, Volume 2, pp. 508-515. IEEE.

Kumar, P., J. Connor, and S. Mikiavcic (2014, Dec). High-throughput 3d reconstruction of plant shoots for phenotyping. In Control Automation Robotics Vision (ICARCV), 2014 13th International Conference on, pp. 211-216.

Li, L., Q. Zhang, and D. Huang (2014). A review of imaging techniques for plant phenotyping. Sensors 14(11), 20078-20111.

Lou, L., Y. Liu, J. Han, and J. Doonan (2014). Accurate multi-view stereo 3D reconstruction for cost-effective plant phenotyping. In A. Campilho and M. Kamel (Eds.), Image Analysis and Recognition, Volume 8815 of Lecture Notes in Computer Science, pp. 349-356. Springer International Publishing.

Meyer, G. E. and J. C. Neto (2008). Verification of color vegetation indices for automated crop imaging applications. Computers and Electronics in Agriculture 63(2), 282-293.

Ohtake, Y., A. Belyaev, M. Alexa, G. Turk, and H.-P. Seidel (2005). Multi-level partition of unity implicits. In SIGGRAPH 2005, SIGGRAPH '05, New York, NY, USA. ACM.

Paproki, A., X. Sirault, S. Berry, R. Furbank, and J. Fripp (2012). A novel mesh processing based technique for 3d plant analysis. BMC Plant Biology 12(1).

Rusu, R. and S. Cousins (2011, May). 3D is here: Point cloud library (pcl). In Robotics and Automation (ICRA), 2011 IEEE International Conference on, pp. 1-4.

Sabater, N., J.-M. Morel, and A. Almansa (2011, March). How accurate can block matches be in stereo vision? SIAM J. Img. Sci. 4(1), 472-500. 